Harold Zirin, Gwaxiang Ai, and Haimin Wang (eds.)

\title{
THE DISTRIBUTION OF SUNSPOT DECAY RATES
}

\author{
V. MARTÍNEZ PILLET, F. MORENO-INSERTIS, M. VÁZQUEZ \\ Instituto de Astrofísica de Canarias, 38200 La Laguna, Tenerife, Spain
}

\begin{abstract}
The distribution of sunspot decay rates is studied using the Greenwich Photoheliographic Results (GPR). The decay rates are seen to be lognormally distributed. An analysis of the lognormal distributions associated to each sunspot group type and for isolated spots is presented. As a remarkable property of the decay process, we show that it happens at a constant total to umbral area ratio.
\end{abstract}

\section{INTRODUCTION}

The distribution of sunspot decay rates is studied using the Greenwich Photoheliographic Results (GPR). We shall distinguish between La Laguna type 3 groups (groups that end as isolated spots, typically Zürich $\mathrm{H}$ and $\mathrm{J}$ ) and La Laguna type 2 groups (which are always complex groups with more than one spot, typically Zürich D, E, F and G). For the period from 1940 to 1976 we have available the more detailed Zürich classification, from where a set of truly "isolated spots" is defined. Sometimes, we also classify the groups as recurrent and non-recurrent ones.

\section{DATA SELECTION}

We select for further analysis only those observations satisfying:

a) Umbral areas larger than $15 \mathrm{MSH}$ and total areas exceeding $35 \mathrm{MSH}$.

b) Observations with heliographic longitude less than 60 degrees.

A particular group, or isolated spot, is included in the analysis if it has 5 or more observations satisfying conditions a) and b). For each of these cases a linear and a parabolic least square fit to $A(t)-t$ is made. These laws are suggested from the following hypothese:

i) Constant decay rate (Bumba, 1963; Gokhale and Zwaan, 1972; Meyer et al., 1974) started at time $t_{o}$ :

$$
\frac{d A(t)}{d t}=\text { constant }, \Rightarrow A(t)=D\left(t-t_{o}\right)+A_{o} .
$$

ii) Decay rate proportional to the length of the spot boundary (Simon and Leighton, 1964; Schmidt, 1968):

$$
\frac{d A(t)}{d t}=-m^{2} \sqrt{A(t)}, \Rightarrow A(t)=q\left(t-t_{o}\right)^{2}+l\left(t-t_{0}\right)+A_{o},
$$


with the additional constrains:

$$
q>0, \quad 1=-2 \sqrt{q A_{o}}
$$

\section{RESULTS}

1) The average decay rates of sunspot groups and isolated spots are lognormally distributed. The parameter $D$ of equation (1) is lognormally distributed. The histogram of this quantity is adequately described by a two-parameter lognormal distribution function:

$$
d N(D)=\frac{1}{\sqrt{2 \pi} \sigma_{\log D}} e^{-\frac{\left(\log D-\mu_{\log D}\right)^{2}}{2 \sigma_{\log D}^{2}}} d \log D
$$

so that the quantity $\log D$ is normally distributed with mean $\mu_{\log D}$ and variance $\sigma_{\log D}^{2}$ (see Figure 1). In Table 1 we give, for each set of sunspot groups, the parameters $\mu_{\log D}$ and $\sigma_{\log D}$.

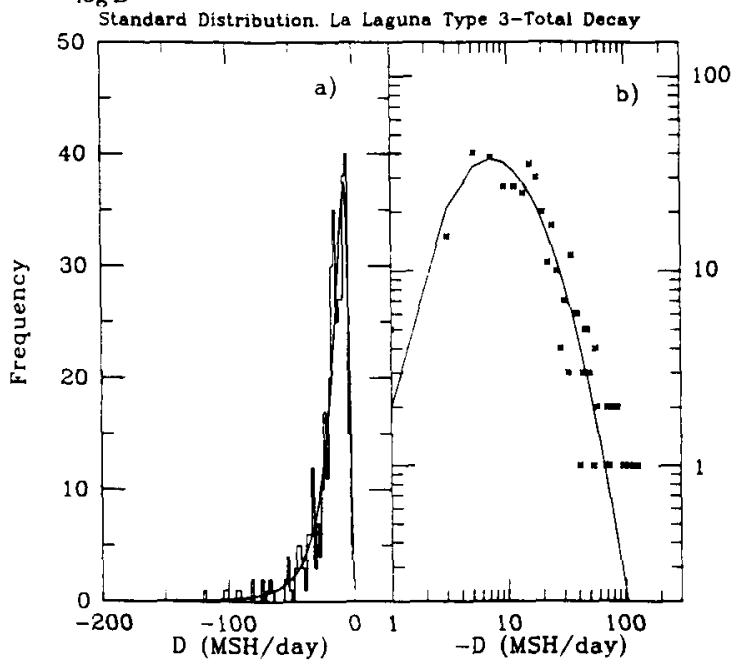

Fig. 1. Comparison between the histogram of decay rates La Laguna type 3 groups total area decay and the predictions of a lognormal function of the type of equation (4).The distribution function is normalized to the same area as the histogram. a) linear scale. b) logarithmic scale.

2) Recently, Howard (1992) has presented the distribution of daily spot group umbral area change. We have analyzed the negative part of his distribution (the "instantaneous" area decay rates) and found that a lognormal description of it is also possible. The resulting parameters were $\mu_{\log } D=1.75$ and $\sigma_{\log D}=1.09$.

3) The lognormal distribution explains the discrepancies found between the decay rates given by different authors. Bumba (1963) obtained, for isolated 
spots, total decay rates of $-4.2 \mathrm{MSH} / \mathrm{day}$, five times lower than those reported by Moreno-Insertis and Vázquez (1988). Bumba's determination was directed towards selecting up the value that appeared most frequently, i.e., the mode of the distribution. Moreno-Insertis and Vázquez (1988), on the other hand, determined the mean value, which differs from the mode in the case of a lognormal distribution.

Table 1

Lognormal Parameters of Sunspot Groups

\begin{tabular}{cccc}
\hline & & & \\
\hline Set $^{1}$ & N. Cases & $\mu_{\log D}$ & $\sigma_{\log D}$ \\
\hline T2 & 515 & 3.373 & 0.869 \\
U2 & 515 & 1.578 & 0.831 \\
T3 & 375 & 2.619 & 0.806 \\
U3 & 375 & 0.957 & 0.784 \\
i-T & 216 & 2.304 & 0.827 \\
i- U & 216 & 0.606 & 0.894 \\
\hline
\end{tabular}

$1 \mathrm{~T}=$ total decay, $\mathrm{U}=$ umbral decay, 2=La Laguna type 2, 3=La Laguna type 3 , $\mathrm{i}-=$ isolated spot.

4) During sunspot decay the ratio between total and umbral areas is constant. We have found that:

$$
r_{D}=\frac{D_{T}}{D_{U}} \approx r_{A}(t)=\frac{A_{T}(t)}{A_{U}(t)} \approx 6 .
$$

This is only possible if:

$$
\frac{d r_{A}(t)}{d t}=0
$$

For the set of isolated spots we have computed the quantity:

$$
\Delta r_{A}=r_{A}\left(t_{0}\right)-r_{A}(\tau)
$$

where $\tau$ is the time corresponding to the last observation. In Figure 2 we present the results of these calculations for the whole sample of isolated spots. This Figure clearly shows that the spots tend to cluster around the zero value of $\Delta r_{A}$ or, in other words, the preference is not to change the $r_{A}$ parameter as the spot dissolves. The spot seems to be adjusting its structure continuously in such a way that the loss of some of its area produces a change in both umbra and penumbra, that maintains this ratio constant.

5) As pointed out by Harvey and Harvey (1973), the net magnetic flux lost from the spot by Moving Magnetic Features (MMF) is similar to the value predicted by the area decay rate. This suggests that the mechanism that destroys the spot works at the boundary of the magnetic rope, i.e., a decay law like equation (2). From the parabolic fits, we have found that the first condition of equation (3) $(q>0)$ is fulfilled by $70-80 \%$ of the sample for all the spot types (including isolated spots). A linear process with random noise would have created an average of $50 \%$, so that positive $q$ seems somewhat favoured. This 
weak non-linearities in the decay phase are more easily detected when several transits are considered. On the other hand, the second condition of equation (3) $\left(l=-2 \sqrt{q A_{o}}\right)$ does not seem to be followed by real spots: The GPR data do not favour the hypothesis of decay with instantaneous rate proportional to the length of the spot boundary. A definite statement about the shape of the decay curve will have to come from the analysis of high-spatial resolution observations of the area and magnetic field disappearance process.

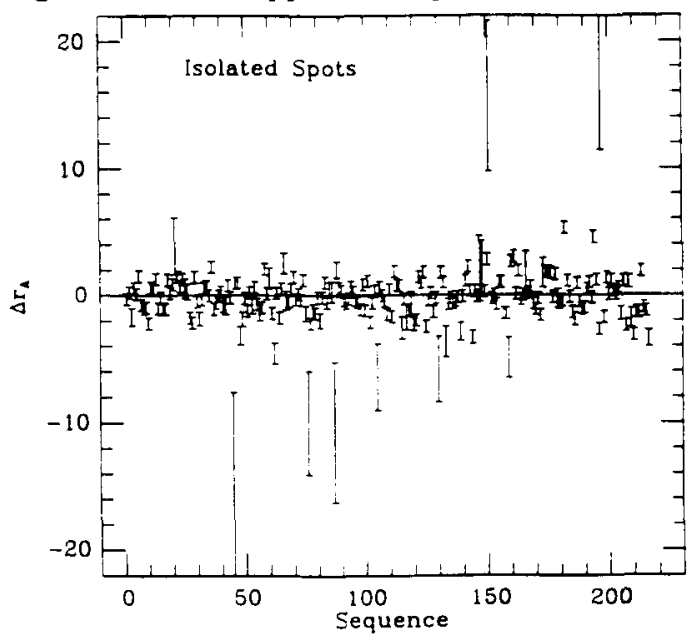

Fig. 2. The change in the $r_{A}$ parameter estimated as the difference between the initial and final values of $r_{A}$ as computed from the linear fits. Data for recurrent and non-recurrent isolated spots are given.

\section{ACKNOWLEDGEMENTS}

This work was partly funded by the DGICYT under project PB91-0530.

\section{REFERENCES}

Bumba, V.: 1963, Bull. astr. Inst. Czech., 14, 91

Gokhale, M.H., and Zwaan, C.: 1972, Solar Phys., 26, 52

Harvey, K., and Harvey, J.: 1973, Solar Phys., 28, 61

Howard, R.F.: 1992, Solar Phys., 137, 51

Meyer, F., Schmidt, H.U., Weiss, N.O., and Wilson, P.R.: 1974, M.N.R.A.S., 169,35

Moreno-Insertis, F., and Vázquez, M.: 1988, Astr. Ap., 205, 289

Schmidt, H.U.: 1968, in K.O. Kipenheuer (ed.), Structure and Development of Solar Active Regions, Reidel, Dordrecht

Simon, G. W., and Leighton, R.B.: 1964, Ap. J., 140, 1120 